\title{
DISTRIBUTION OF POROSITY OF EMULSION PHASE AND ITS EFFECT ON CONVERSION IN A FLUIDIZED BED
}

\author{
MASAO YAMAZAKI, KAZUSHI FUKUTA, YUAN-HENG LI \\ AND JUN-ICHI TOKUMOTO \\ Department of Chemical Engineering, Nagoya University, Nagoya 464
}

Key Words: Fluidization, Chemical Reaction, Chemical Reactor, Conversion, Reversible Reaction, Instrumentation, Porosity Distribution, Bubble Distribution

\begin{abstract}
Spatial distribution of emulsion-phase porosity and bubble frequency in a fluidized bed of cracking catalyst are determined by use of a seven-channel light transmission method developed for this purpose. The bed was operated at moderate gas velocity at atmospheric pressure or at low gas velocity in slightly pressurized conditions.

Conversion was calculated by taking into account the physical properties of the emulsion phase such as porosity, interstitial gas velocity and volume fraction. Experiments revealed in general the non-uniform features within a bed; therefore reactant concentrations and conversions calculated numerically were compared with those from the analytical solutions for an isothermal first-order reversible reaction.

The conversion in the emulsion phase was almost completed at $0.1 \mathrm{~m}$ height above the distributor. Hence, the overall conversion was controlled in the bubble phase. Increasing bed pressure was accompanied by a decrease in the volume fraction of the emulsion phase, and the pressure effect on the overall conversion at the exit of the dense phase under $300 \mathrm{kPa}$, for example, amounted to approximately seven percent gain in conversion in comparison with the case of atmospheric pressure, when other factors such as reaction scheme, reaction rate, gas exchange rate and volume fraction of catalyst in bubble phase were assumed to be independent of pressure.

It was also suggested that the conventional two-phase theory seemed to give approximately $1 \%$ higher conversion than the numerically calculated figure under the same assumptions.
\end{abstract}

\section{Introduction}

There have been some reports on the pressure effect on a bed that dealt with minimum fluidization velocity, ${ }^{2,5,9)}$ bubble frequency ${ }^{1,5)}$ bed voidage and porosity of the emulsion phase. ${ }^{1-3,7,9,13,15)}$ Concerning the porosity of emulsion phase, the authors developed a light transmission method with a bubble elimination circuit which gives the local porosity of emulsion phase with high accuracy. ${ }^{6,10,13)}$ The distribution of porosity of emulsion phase was determined under both a moderate superficial air velocity at atmospheric pressure and a low superficial air velocity in a slightly pressurized fluidized bed, assuming the operating pressure of the Sohio process for acrilonitrile or of the oxychlorination process for ethylene dichloride.

The characteristics of emulsion phase such as porosity distribution and local interstitial gas velocity were considered, and conversion was estimated on the basis of the proposed reaction equations with the obtained data of emulsion phase and other empirical results on gas exchange rate..$^{11,12}$

Received August 2, 1986. Correspondence concerning this article should be addressed to M. Yamazaki. K. Fukuta is now with Brother Industries, Ltd., Nagoya 467. Y. H $\mathrm{Li}$ is now with Zhalainour Mining Administration Bureau, Inner Mongolian Autonomous Region, The People's Republic of China. J. Tokumoto is now with NTK Spark Plug Co., Ltd., Nagoya 467.

\section{Theoretical}

The authors developed a modified reaction equation in the emulsion phase for an isothermal firstorder reversible process in a fluidized bed, taking into account a correction factor $\gamma$, volume fraction $\phi_{e}$ and superficial local gas velocity $u_{e} \phi_{e}$ in that phase. The following are the equations, together with that for the bubble phase. ${ }^{10)}$

Bubble phase:

$$
\begin{aligned}
& -\frac{d A_{b}}{d\left(z / L_{f}\right)}-\frac{k_{o b} a_{b} L_{f}}{u_{b} \phi_{b}}\left(A_{b}-A_{e}\right)-\frac{\mu \phi_{b} k_{r} L_{f}}{u_{b} \phi_{b}}\left(A_{b}-\frac{P_{b}}{K}\right)=0 \\
& -\frac{d P_{b}}{d\left(z / L_{f}\right)}-\frac{k_{o b} a_{b} L_{f}}{u_{b} \phi_{b}}\left(P_{b}-P_{e}\right)+\frac{\mu \phi_{b} k_{r} L_{f}}{u_{b} \phi_{b}}\left(A_{b}-\frac{P_{b}}{K}\right)=0
\end{aligned}
$$

Emulsion phase:

$$
\begin{gathered}
-\frac{d A_{e}}{d\left(z / L_{f}\right)}+\frac{k_{o b} a_{b} L_{f}}{u_{e} \phi_{e}}\left(A_{b}-A_{e}\right)-\frac{\gamma \phi_{e} k_{r} L_{f}}{u_{e} \phi_{e}}\left(A_{e}-\frac{P_{e}}{K}\right)=0 \\
-\frac{d P_{e}}{d\left(z / L_{f}\right)}+\frac{k_{o b} a_{b} L_{f}}{u_{e} \phi_{e}}\left(P_{b}-P_{e}\right)+\frac{\gamma \phi_{e} k_{r} L_{f}}{u_{e} \phi_{e}}\left(A_{e}-\frac{P_{e}}{K}\right)=0
\end{gathered}
$$

Here, $A$ and $P$ are reactant and product con- 
Table 1. Summary of solutions when $A_{b o}=A_{e o}$

$$
\begin{aligned}
& A_{b}(Z)=A_{b o}\left(\frac{1}{K+1}\right)\left[1+K\left\{e^{\beta Z}+\delta\left(e^{\alpha Z}-e^{\beta Z}\right)\right\}\right] \\
& A_{e}(Z)=A_{b o}\left(\frac{1}{K+1}\right)\left[1+\left(\frac{K}{N_{o b}}\right)\left(\left\{\beta+N_{o b}+\left(1+\frac{1}{K}\right) N_{b r}\right\} e^{\beta Z}+\delta\left[\left\{\alpha+N_{o b}+\left(1+\frac{1}{K}\right) N_{b r}\right\} e^{\alpha Z}-\left\{\beta+N_{o b}+\left(1+\frac{1}{K}\right) N_{b r}\right\} e^{\beta Z}\right]\right)\right] \\
& P_{b}(Z)=A_{b o}\left(\frac{K}{K+1}\right)\left[1-\left\{e^{\beta Z}+\delta\left(e^{\alpha Z}-e^{\beta Z}\right)\right\}\right] \\
& P_{e}(Z)=A_{b o}\left(\frac{K}{K+1}\right)\left[1-\left(\frac{1}{N_{o b}}\right)\left(\left\{\beta+N_{o b}^{*}+\left(1+\frac{1}{K}\right) N_{b r}\right\} e^{\beta Z}+\delta\left[\left\{\alpha+N_{o b}+\left(1+\frac{1}{K}\right) N_{b r}\right\} e^{\alpha Z}-\left\{\beta+N_{o b}+\left(1+\frac{1}{K}\right) N_{b r}\right\} e^{\beta Z}\right]\right)\right]
\end{aligned}
$$

where

$$
\begin{aligned}
& \alpha=-\frac{1}{2}\left\{\left(N_{o b}+N_{e o b}\right)+\left(1+\frac{1}{K}\right)\left(N_{b r}+N_{e r}\right)\right\}+\frac{1}{2} \sqrt{D} \\
& \delta=-\frac{1}{\sqrt{D}}\left\{\beta+\left(1+\frac{1}{K}\right) N_{b r}\right\} \\
& \beta=-\frac{1}{2}\left\{\left(N_{o b}+N_{e o b}\right)+\left(1+\frac{1}{K}\right)\left(N_{b r}+N_{e r}\right)\right\}-\frac{1}{2} \sqrt{D} \\
& D=\left\{\left(N_{o b}-N_{e a b}\right)+\left(1+\frac{1}{K}\right)\left(N_{b r}-N_{e r}\right)\right\}^{2}+4 N_{o b} N_{e o b}
\end{aligned}
$$

centrations, respectively, $z$ is the height above the distributor, $L_{f}$ is the fluidized bed height, and $K$ is the ratio of rate constant of forward reaction to that of backward reaction where subscripts $b$ and $e$ are for bubble and emulsion phases, respectively.

$\gamma$ in Eqs. (3) and (4) is a correction factor that reduces emulsion phase to its equivalent fixed bed whose porosity is $\varepsilon_{o}$, and is defined as follows:

$$
\gamma=\frac{1-\left\langle\varepsilon_{e m l}\right\rangle}{1-\varepsilon_{o}}
$$

where $\varepsilon_{e m l}$ is the local porosity of emulsion phase and $\left\langle\varepsilon_{e m l}\right\rangle$, which will be discussed in detail later, is its ensemble average at $z$. The factor $\gamma$ should be included in the reaction equation of emulsion phase if the reaction rate constant is determined on the fixed-bed basis. Equations (1)-(4) are rewritten in dimensionless form as

$$
\begin{aligned}
& -\frac{d A_{b}}{d Z}-N_{o b}\left(A_{b}-A_{e}\right)-N_{b r}\left(A_{b}-\frac{P_{b}}{K}\right)=0 \\
& -\frac{d P_{b}}{d Z}-N_{o b}\left(P_{b}-P_{e}\right)+N_{b r}\left(A_{b}-\frac{P_{b}}{K}\right)=0 \\
& -\frac{d A_{e}}{d Z}+N_{e o b}\left(A_{b}-A_{e}\right)-N_{e r}\left(A_{e}-\frac{P_{e}}{K}\right)=0 \\
& -\frac{d P_{e}}{d Z}+N_{e o b}\left(P_{b}-P_{e}\right)+N_{e r}\left(A_{e}-\frac{P_{e}}{K}\right)=0
\end{aligned}
$$

When the bed characteristics can be assumed uniform throughout the bed, the four dimensionless numbers in the basic equations become constant and Eqs. $(1)^{\prime}-(4)^{\prime}$ can be solved analytically under the following initial conditions. ${ }^{8,10}$ )

$$
Z=0 ; \quad A_{b}(Z)=A_{b o}, \quad P_{b}(Z)=0
$$

$$
Z=0 ; \quad A_{e}(Z)=A_{e o}, \quad P_{e}(Z)=0
$$

where $A_{b o}$ and $A_{e o}$ are the inlet reactant concentrations.

The reactant would usually be fed into the fluidizing medium, so the inlet reactant concentrations in both phases would be equal, i.e., $A_{b o}=A_{e o}$. The analytical solutions in that case are summarized in Table 1. ${ }^{10 \text { ) }}$

Obviously, Eqs. (8)-(15) are all functions of $N_{e r}$, which is also a function of $\gamma$ and thus of $\varepsilon_{e m l}$.

From Eqs. (8) and (9), three kinds of conversions $X_{b}, X_{e}$ and $X$ can be defined in general form as follows:

$$
\begin{aligned}
& X_{b}(Z)=1-\frac{A_{b}(Z) u_{b} \phi_{b}}{A_{b o}\left(u_{b} \phi_{b}\right)_{o}} \\
& X_{e}(Z)=1-\frac{A_{e}(Z) u_{e} \phi_{e}}{A_{e o}\left(u_{e} \phi_{e}\right)_{o}} \\
& X(Z)=1-\frac{A_{b}(Z) u_{b} \phi_{b}+A_{e}(Z) u_{e} \phi_{e}}{A_{b o}\left(u_{b} \phi_{b}\right)_{o}+A_{e o}\left(u_{e} \phi_{e}\right)_{o}}
\end{aligned}
$$

where $(u \phi)_{o}$ is the inlet superficial gas velocity, $X_{b}$ is the conversion in bubble phase, $X_{e}$ is that in emulsion phase and $X$ is the overall conversion.

When the bed characteristics are uniform and if the reactant is fed into the fluidizing medium, the following relation hold:

$$
\begin{aligned}
& \left(u_{b} \phi_{b}\right)_{o}=u_{b} \phi_{b}=\mathrm{const} . \\
& \left(u_{e} \phi_{e}\right)_{o}=u_{e} \phi_{e}=\mathrm{const} .
\end{aligned}
$$

Then Eqs. (16)-(18) reduce to the conventional forms below, since $A_{b o}=A_{e o}$.

$$
X_{b}(Z)=1-\frac{A_{b}(Z)}{A_{b o}}
$$




$$
\begin{aligned}
& X_{e}(Z)=1-\frac{A_{e}(Z)}{A_{e o}} \\
& X(Z)=1-\frac{A_{b}(Z) u_{b} \phi_{b}+A_{e}(Z) u_{e} \phi_{e}}{A_{b o} u_{f}}
\end{aligned}
$$

In the course of the conversion calculation, the following subsidiary equations based on the conventional two-phase theory are also requisite:

$$
\begin{gathered}
\phi_{b}+\phi_{e}=1 \\
u_{b} \phi_{b}+u_{e} \phi_{e}=u_{f}
\end{gathered}
$$

where $\phi_{b}$ is measured by the proposed light transmission method. As for $u_{e} \phi_{e}$, the empirical equation below is assumed to hold:

$$
\begin{aligned}
u_{e} \phi_{e}(Z) & \doteqdot u_{m b}(2-Z) \\
& \doteqdot 3 u_{m f}(2-Z)
\end{aligned}
$$

which was obtained by a method using both a pressure transducer and a light transmission probe in a bed of cracking catalyst. ${ }^{4)}$ There is a small amount of air flow, about twice $u_{m b}$ in the lower part of the bed $(1 \gg Z)$ and $u_{m b}$ in the upper part $(Z \doteqdot 1)$ where $u_{m b}$ is nearly equal to thrice $u_{m f}$. The gas exchange rate $k_{o b} a_{b}$ in $N_{o b}$ and $N_{e o b}$ can be estimated by the following modified correlations, obtained by experiments in heat transfer: ${ }^{1,12)}$

$$
\frac{k_{o b} a_{b}}{\phi_{b}}=28 u_{f}^{1 / 2}
$$

or

$$
\frac{k_{o b} a_{b}}{\phi_{b}}=25.2 u_{f}^{0.76}
$$

which are a semi-theoretical and an empirical equation, respectively. Hence, the four $N_{s}^{\prime}$ in Eqs. (8), (9), (16)-(18) and (19)-(21) are all to be determined by knowing $\phi_{b}$ and $\varepsilon_{e m l}$ (or $\gamma$ ).

\section{Experimental}

Figure 1 is an outline of the experimental apparatus, which can be pressurized up to $400 \mathrm{kPa}$ and is operated at low superficial air velocity. A column of steel (9) ( $82 \mathrm{~mm}$ i.d. $\times 1 \mathrm{~m}$ high) is charged with cracking catalyst $\left(\bar{d}_{p}=67 \mu \mathrm{m}, \rho_{s}=830 \mathrm{~kg} \cdot \mathrm{m}^{-3}, \varepsilon_{0}=0.365\right.$, $\left.\varepsilon_{m f}=0.43, u_{m f}=0.0016 \mathrm{~m} \cdot \mathrm{s}^{-1}\right)$ of weight $W(=1.6 \mathrm{~kg})$ at static bed height $L_{s}(=0.62 \mathrm{~m})$. Distributor (7) is a perforated brass plate $1.25 \mathrm{~mm}$ thick with $1 \mathrm{~mm}$ diameter holes, located in a staggered arrangement of $2 \mathrm{~mm}$ pitch and 25 holes $\cdot \mathrm{cm}^{-2}$. Calming section (6) is a packed bed of glass beads ( $5 \mathrm{~mm}$ diameter), $0.1 \mathrm{~m}$ high. Probe (8) is a linear arrangement of seven pairs of elements hanging along a strip $(0.7-0.8 \mathrm{~mm}$ thickness $\times 5 \mathrm{~mm}$ height $\times 82 \mathrm{~mm}$ length) of epoxy resin. Another column of plexiglass of the same size with an

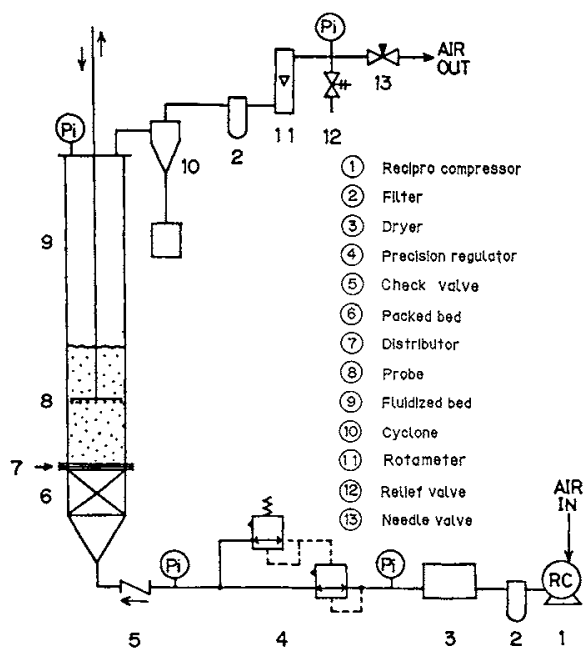

Fig. 1. Schematic diagram of fluidized bed system which can be pressurized up to $400 \mathrm{kPa}$

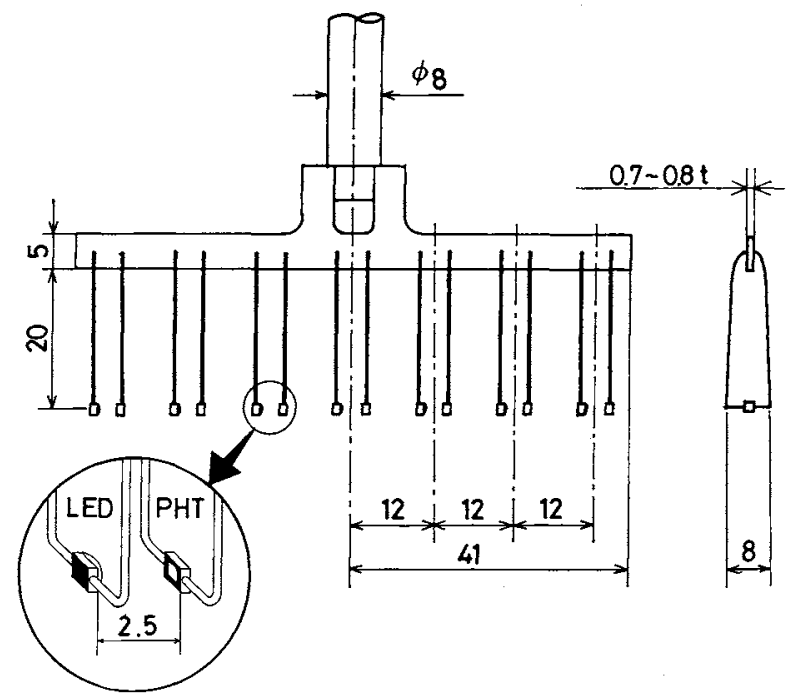

Fig. 2. Details of a linear light transmission probe. Seven pairs of elements are hung along a strip of epoxy resin at 12$\mathrm{mm}$ pitch

enlarged section at its top is used for the run at moderate superficial air velocity at atmospheric pressure.

Figure 2 shows details of the probe framework, in which the elements of each pair are facing each other $2.5 \mathrm{~mm}$ apart and seven such pairs are arrayed at $12 \mathrm{~mm}$ pitch. The probe is attached to the tip of a stainless steel tube ( $8 \mathrm{~mm}$ o.d.) and can occupy almost any place in the bed as the tube moves up and down or rotates.

In the experiments, the tube was rotated by $\theta$ or 60 degrees twice at a certain level. As the off-centered six elements of the probe were set in three directions, so nineteen spots were selected in all including the center for measurement at a given level $z$. Such a procedure is sketched in Fig. 3.

Figure 4 is a block diagram of the light transmission 
method, which is an extension of the previous method. ${ }^{6,13)}$ The dotted block denotes the probe system containing seven pairs of sensors. Each pair of sensors is made of a light source (an infrared light-emitting diode) and a photo-transistor detector which is adjusted to produce $10 \mathrm{v}$ maximum when an ideally clean bubble should happen to come. The seven channels' photo currents are converted to voltage via currentto-voltage converters and multiplexed with the analog

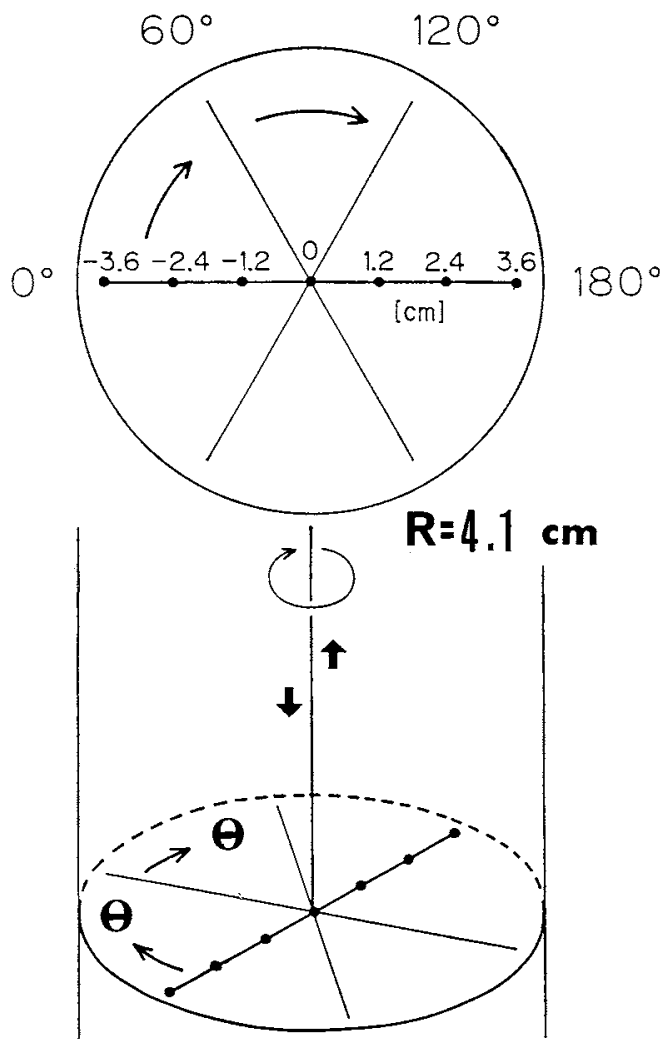

Fig. 3. The probe stem can move up and down, or rotate. Location of the probe elements after the stem is twice rotated $60^{\circ}$ at a given level multiplexer. Each channel can be selected manually or switched automatically at $75 \mathrm{~Hz}$. The output from the multiplexer is transferred to the analog switch where bubble signals are eliminated and a pure emulsion signal is extracted. The emulsion signal is timeaveraged by an integrator for a certain measuring term $T$ per run. Usually $T$ is set for $100 \mathrm{~s}$ and repeated twice.

Bubble frequency $f_{b}$ is the number of bubbles during $T$ and bubble fraction $\phi_{b}$ is the total number of bubble duration terms during $T$. Those three numerical values are obtained by three counters.

The porosity of emulsion phase $\varepsilon_{e m l}$ is determined as shown below, assuming the Lambert-Beer law.

$$
\begin{gathered}
E_{i}=E_{a} e^{-\kappa \rho_{i}} \\
\bar{E}_{e}=\frac{1}{R C} \int_{0}^{T_{e}} E_{e} d t
\end{gathered}
$$

where $E_{i}$ is the output of the analog multiplexer, $E_{e}$ that of analog switch, $\kappa$ a constant related to the extinction coefficient of a medium and $\rho_{i}$ is the apparent density of a medium or the emulsion phase. Meanwhile the probe is dipped within a clean bubble, $E_{i}$ becomes identical to $E_{a}$, which is first adjusted to $10.0 \mathrm{v}$ in clean static air $\left(\rho_{i}=\rho_{\text {air }} \doteqdot 0 \mathrm{~kg} \cdot \mathrm{m}^{-3}\right)$ in the dark. $\bar{E}_{e}$ is the time average of $E_{e}$ defined by Eq. (28), where $R C(=33.3 \mathrm{~s})$ is the integral time of the integrator and $T_{e}$ is the emulsion term free of bubbles. From Eqs. (27) and (28), $\kappa$ of a pair of sensors is given by measuring $E_{r}$ when $\rho_{r}$, the apparent density of material of a reference bed, is known:

$$
\begin{aligned}
\kappa & =-\frac{1}{\rho_{r}} \ln \left(\frac{E_{r}}{E_{a}}\right) \\
& =-\frac{1}{\rho_{r}} \ln \left(\frac{R C \bar{E}_{r}}{T E_{a}}\right)
\end{aligned}
$$

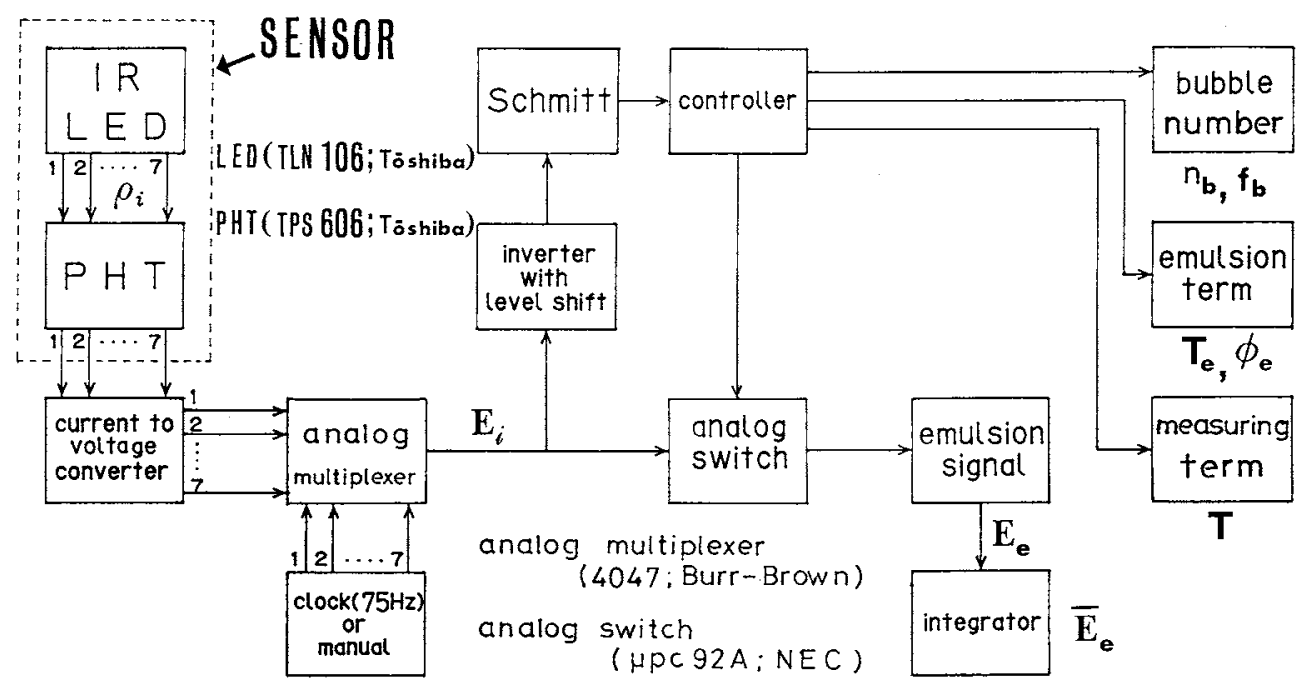

Fig. 4. Block diagram of extended light transmission method (seven channels) 


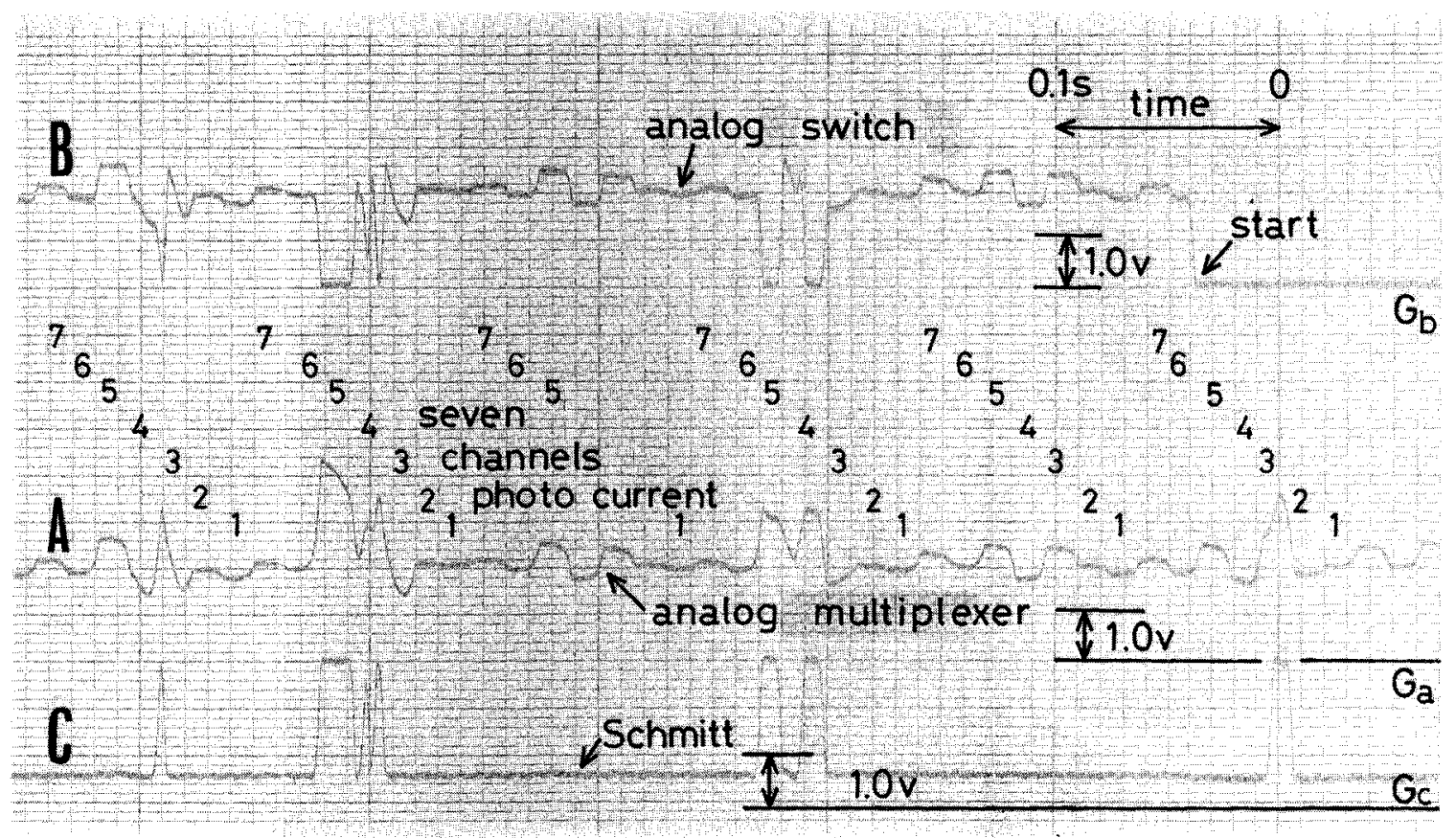

Fig. 5. Oscillogram of photo-currents and bubbles among seven channels when switching at $75 \mathrm{~Hz}$ (cracking catalyst, $D_{T}=82 \mathrm{~mm}$ i.d., $P=200 \mathrm{kPa}, L_{s}=0.62 \mathrm{~m}, u_{f}=0.03 \mathrm{~m} \cdot \mathrm{s}^{-1}$ )

The seven $\kappa$-values determined by the above method range from ca. 4.0 to 6.0. $\rho_{i}$ and hence $\varepsilon_{i}$ can be determined by the following equations, using both $\kappa$ and the measured $\bar{E}_{e}$.

$$
\begin{aligned}
\rho_{i} & =-\frac{1}{\kappa} \ln \left(\frac{R C \bar{E}_{e}}{T_{e} E_{a}}\right) \\
\varepsilon_{i} & =1-\frac{\rho_{i}}{\rho_{s}}
\end{aligned}
$$

where $\rho_{s}$ is the particle density and $830 \mathrm{~kg} \cdot \mathrm{m}^{-3}$ is adopted for cracking catalyst including micropores.

Figure 5 is an oscillogram of three typical signals in the circuit of Fig. 4. Signal $A$ is the output of the multiplexer driven at $75 \mathrm{~Hz}$ and the intervals marked 1-7 correspond to the outputs of detectors 1-7, respectively. The threshold of the Schmitt circuit is set approximately at $2.1 \mathrm{v}$ and whenever the original photo signal $A$ exceeds $2.1 \mathrm{v}$ it is immediately brought down to the ground level. $G$ for that bubble duration, as signal $B$ in the figure clearly shows. Signal $C$ is the output of the Schmitt circuit, which is supposed to be a square pulse train of $2.8 \mathrm{v}$ peak corresponding to each bubble.

\section{Results}

Experiments were done by changing the bed pressure $P$ and superficial velocity of air $u_{f}$ in the bed of cracking catalyst. Figure 6 shows the volume fraction of both phases against $z$ under various pressures. Since $\phi_{b}$ is measured, $\phi_{e}$ is calculated by Eq. (22).

Figure 7 shows the results for the distribution of $\varepsilon_{e m l}$

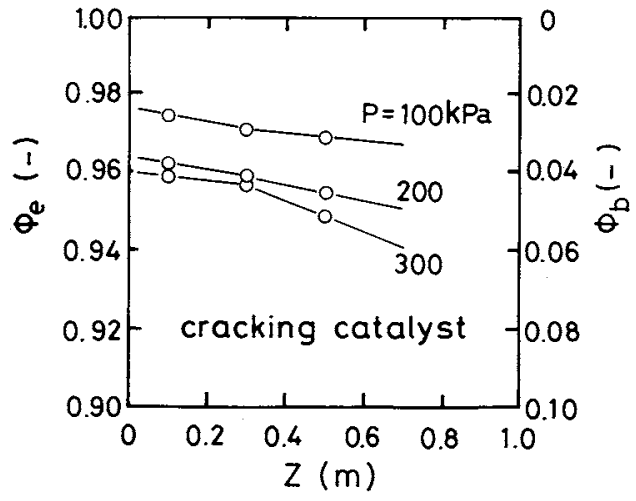

Fig. 6. Fraction of emulsion or bubble phase against $z$ under various pressures (cracking catalyst, $D_{T}=82 \mathrm{~mm}$ i.d., $L_{s}=0.62 \mathrm{~m}, u_{f}=0.05 \mathrm{~m} \cdot \mathrm{s}^{-1}$ )

and $f_{b}$ at six levels $(z=0.05,0.10,0.20,0.30,0.50$ and $0.65 \mathrm{~m})$ under a moderate $u_{f}\left(=0.20 \mathrm{~m} \cdot \mathrm{s}^{-1}\right)$ in an atmospheric fluidized bed $(P=100 \mathrm{kPa})$. The keys in the figures are made clear by referring to Fig. 3 . Both $\varepsilon_{e m l}$ and $f_{b}$ distributions are not as symmetrical as might be expected; some show conspicuous asymmetry under these conditions.

Figures 8A-8D show the results at four levels $(z=$ $0.05,0.10,0.30$ and $0.50 \mathrm{~m})$ under a low $u_{f}$ $\left(=0.05 \mathrm{~m} \cdot \mathrm{s}^{-1}\right)$ in a slightly pressurized fluidized bed $(P=100,200$ and $300 \mathrm{kPa})$. Those results may be considered almost axi-symmetrical, except for a few. During the course of measurement, the element at the axis $(r=0)$ obtains data three times when the strip is rotated by $60^{\circ}$ twice. In the meantime, three axisymmetrical pairs of detectors at $r(\neq 0)$ tagged along 

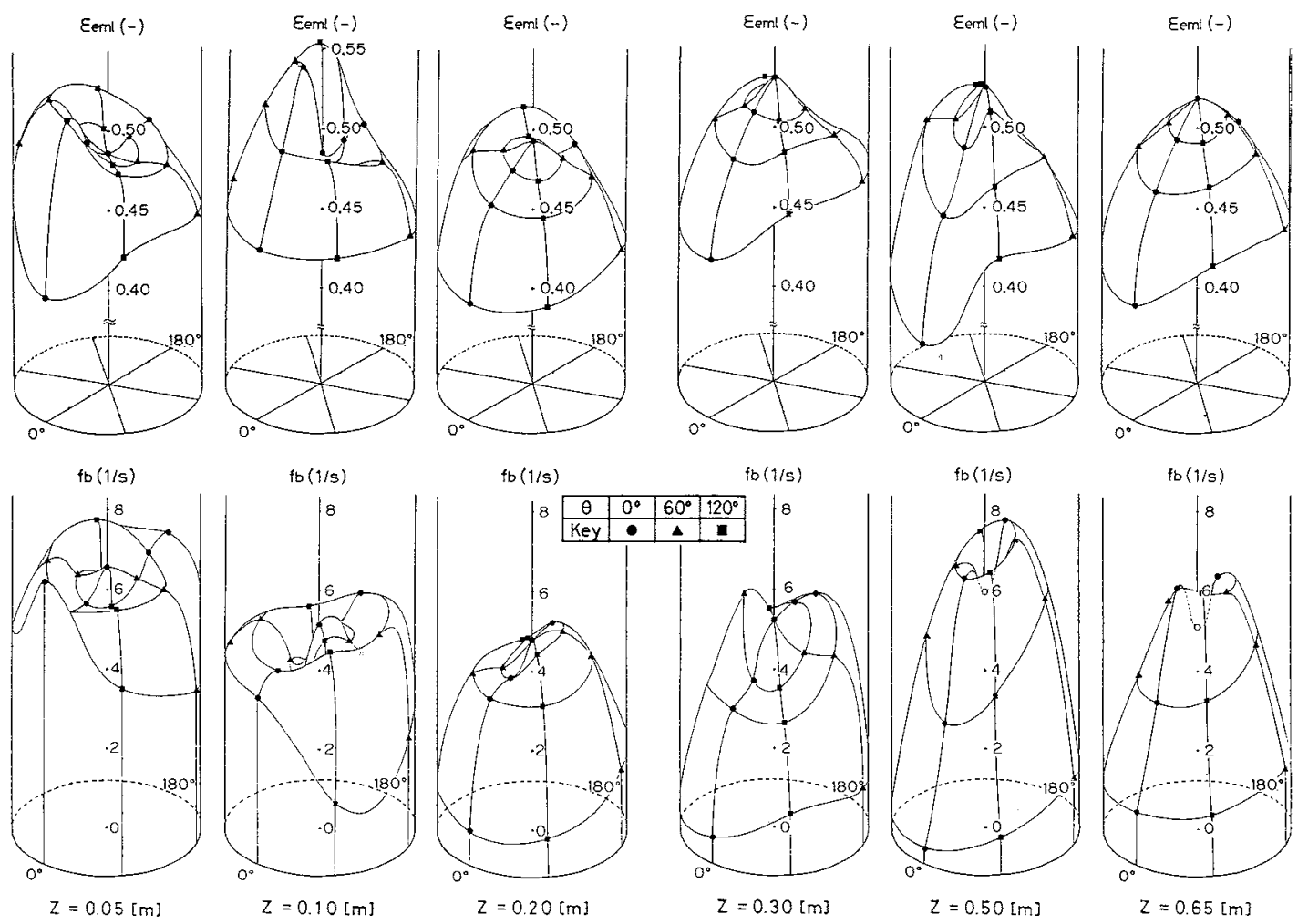

Fig. 7. Distributions of $\varepsilon_{e m l}$ and $f_{b}$ at various $z$ in an atmospheric fluidized bed of plexiglass column (cracking catalyst, $D_{T}=82 \mathrm{~mm}$ i.d., $P=100 \mathrm{kPa}, L_{s}=0.48 \mathrm{~m}, u_{f}=0.20 \mathrm{~m} \cdot \mathrm{s}^{-1}$ )

the strip can obtain data at eighteen points in all on three virtual circumferences, as Fig. 3 shows.

Figure 9 are three-dimensional representations of the mean of porosity of the emulsion phase $\bar{\varepsilon}_{e m l}$ and that of bubble frequency $\bar{f}_{b}$ against $r$ and $z$. Both $\bar{\varepsilon}_{e m l}$ and $\bar{f}_{b}$ at $r(\neq 0)$ are averages of six data and those at $r=0$ are averages of three data. The sense of $z$ coordinate in $\bar{\varepsilon}_{e m l}$-distribution is drawn in reverse to that in $\bar{f}_{b}$-distribution for convenience of inspection.

Since $\varepsilon_{e m l}$ or $\bar{\varepsilon}_{e m l}$ is a function of both $r$ and $z$, a mean is defined to eliminate the variable $r .\left\langle\varepsilon_{e m l}\right\rangle$ in Eq. (5) is deduced as the area weighted mean of $\bar{\varepsilon}_{e m l}$ at $z$, i.e., the product sum of $\left(\bar{\varepsilon}_{e m l}\right)_{i}$ and $s_{i}$ which are the areas of a circle of radius $0.6 \mathrm{~cm}$ and its three concentric rings of width $1.2 \mathrm{~cm}$ :

$$
\left\langle\varepsilon_{e m l}\right\rangle=\frac{1}{S_{i}} \sum_{i=1}^{4}\left(\bar{\varepsilon}_{e m l}\right)_{i}\left(s_{i}\right)
$$

where $S=\sum_{i=1}^{4} s_{i}\left(=52.2 \mathrm{~cm}^{2}\right)$ is the cross section of the
bed.

\section{Discussion}

The relatively low $\bar{f}_{b}$-value at $z=5 \mathrm{~cm}$ inFig. 9 is supposed to be due to a small amount of gas in the emulsion phase which would be squeezed out by particle motion just above the distributor. In Figs. 8 and 9 , the depression of $\bar{\varepsilon}_{e m l}$ at $r=1.2 \mathrm{~cm}$ may suggest that there is a local down-flow of particles where the squeezed gas penetrates toward the center or toward the wall.

In general, the characteristics of a fluidized bed might be treated as being distributed non-uniformly as Fig. 7 shows. In that case, $A_{b}$ and $A_{e}$, and hence $X^{\prime} s$, should be calculated numerically. From Eqs. (25) and (26), the $\left(k_{o b} a_{b} / \phi_{b}\right)$ value at $u_{f}=0.05 \mathrm{~m} \cdot \mathrm{s}^{-1}$ is estimated to be ca. $4.0 \mathrm{~s}^{-1}$ when $P=100 \mathrm{kPa}$. Another factor $\mu$, i.e., volume fraction of catalyst in bubble phase, is assumed to be ca. 0.01 .

Figure $\mathbf{1 0}$ is the calculated result of three kinds of conversion by the Runge-Kutta method for an isothermal first-order reversible reaction when $k_{r}=1.0$ $\mathrm{s}^{-1}, k_{r}^{\prime}=0.5 \mathrm{~s}^{-1}$ and $P=300 \mathrm{kPa}$ using experimental results of $\phi_{e}$ in Fig. 6 and $\left\langle\varepsilon_{e m l}\right\rangle$ from Figs. 8 or 9, as $\phi_{e}$ and $\left\langle\varepsilon_{e m l}\right\rangle$ are both functions of $z$ only. In the calculation, the values of $\phi_{e}$ and $\left\langle\varepsilon_{e m l}\right\rangle$ are extrapolated or interpolated by Simpson's formula from bottom $(z=0)$ to top $\left(z=L_{f}=70 \mathrm{~cm}\right)$ with $z$-increment of $1 \mathrm{~cm}$. Conversion for emulsion phase is almost completed by $z=0.10 \mathrm{~m}$ as $X_{e}$ in Fig. 10 clearly shows. The figure also shows that the overall conversion $X$ would be controlled by bubble phase because the amount of interstitial gas is small.

Figure 11 is the result of overall conversions for irreversible or reversible first-order reaction under slightly pressurized conditions. The improvement of conversion at the top of the dense phase is shown to be $11 \%$ for irreversible reaction and $7 \%$ for reversible 

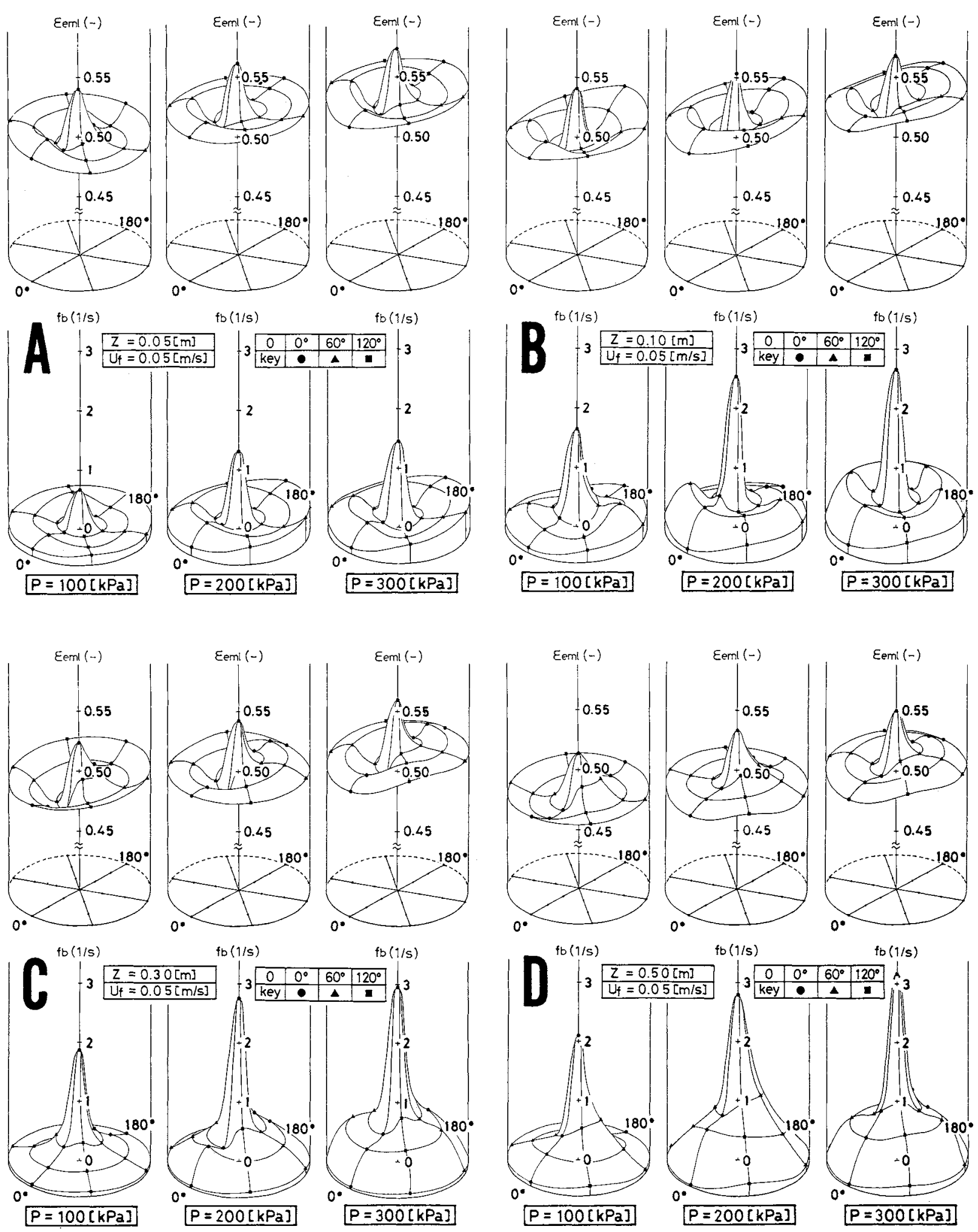

Fig. 8. Distributions of $\varepsilon_{e m l}$ and $f_{b}$ at various $z$ in a slightly pressurized fluidized bed of steel column (cracking catalyst, $D_{T}=82 \mathrm{~mm}$ i.d., $P=100,200$ and $300 \mathrm{kPa}, L_{s}=0.62 \mathrm{~m}, u_{f}=0.05 \mathrm{~m} \cdot \mathrm{s}^{-1}$ )

reaction when the pressure is raised to $300 \mathrm{kPa}$ from atmospheric under the assumption that the pressure effects on $k_{o b} a_{b}, k_{r}, k_{r}^{\prime}$ and $\mu$ are negligible.

The conversion of bubble phase by the conventional two-phase model, shown by the dotted line in
Fig. 12, is calculated by Eqs. (8) and (19) using $u_{f}=0.05 \mathrm{~m} \cdot \mathrm{s}^{-1}, u_{e} \phi_{e}=u_{m b}=0.48 \mathrm{~cm} \cdot \mathrm{s}^{-1}, \phi_{b}=0.05$, $\left\langle\varepsilon_{e m l}\right\rangle=\varepsilon_{e m l}=\varepsilon_{m b}=0.480$ and thus $\gamma=(1-0.480) /$ $(1-0.365)=0.82$. It is approximately $1 \%$ higher than the solid line calculated numerically near the 

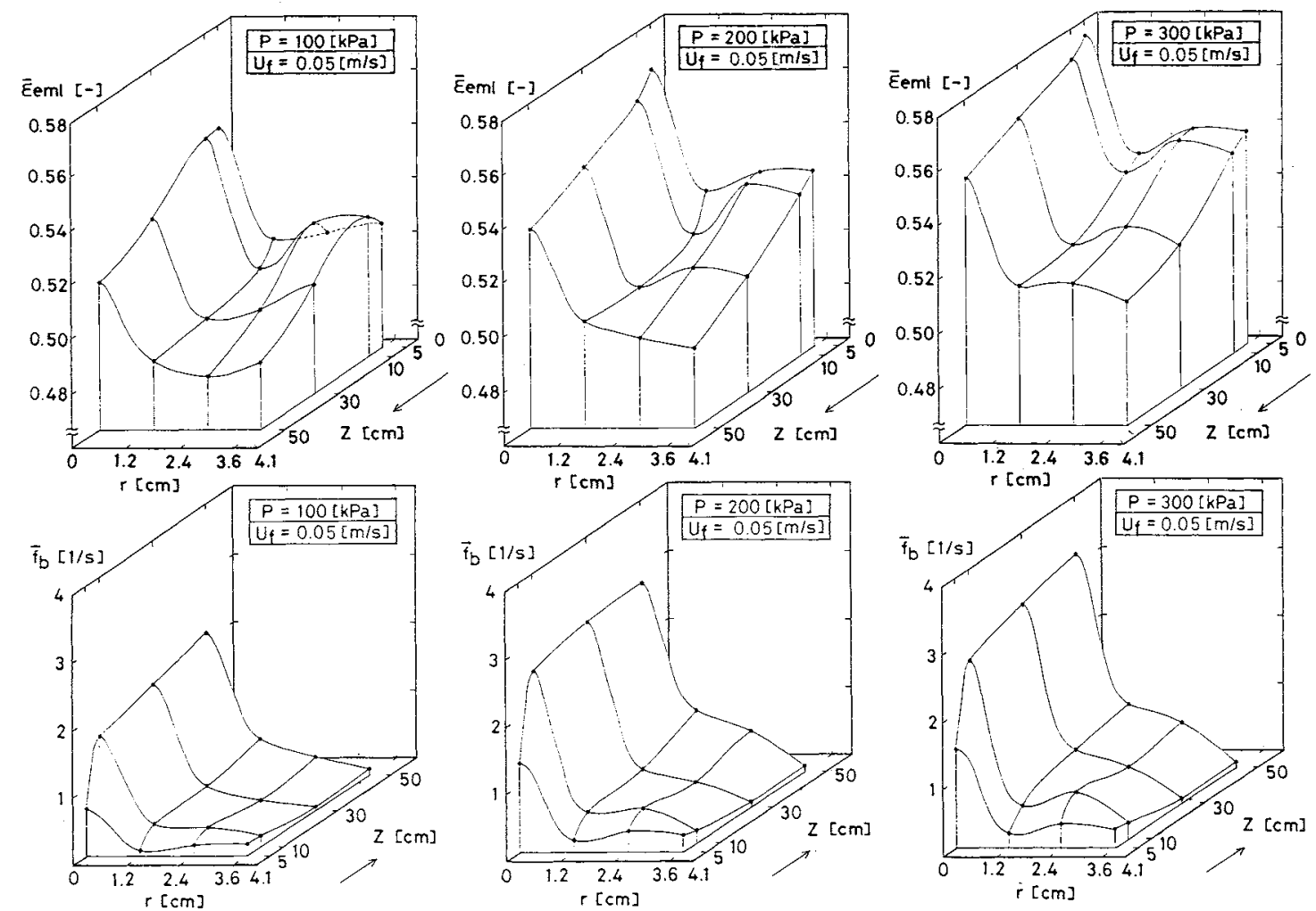

Fig. 9. Mean of $\varepsilon_{\text {eml }}$ and that of $f_{b}$ represented against $r$ and $z$ in a slightly pressurized fluidized bed (cracking catalyst, $D_{T}=82 \mathrm{~mm}$ i.d., $P=100,200$ and $300 \mathrm{kPa}, L_{s}=0.62 \mathrm{~m}, u_{f}=0.05 \mathrm{~m} \cdot \mathrm{s}^{-1}$ )

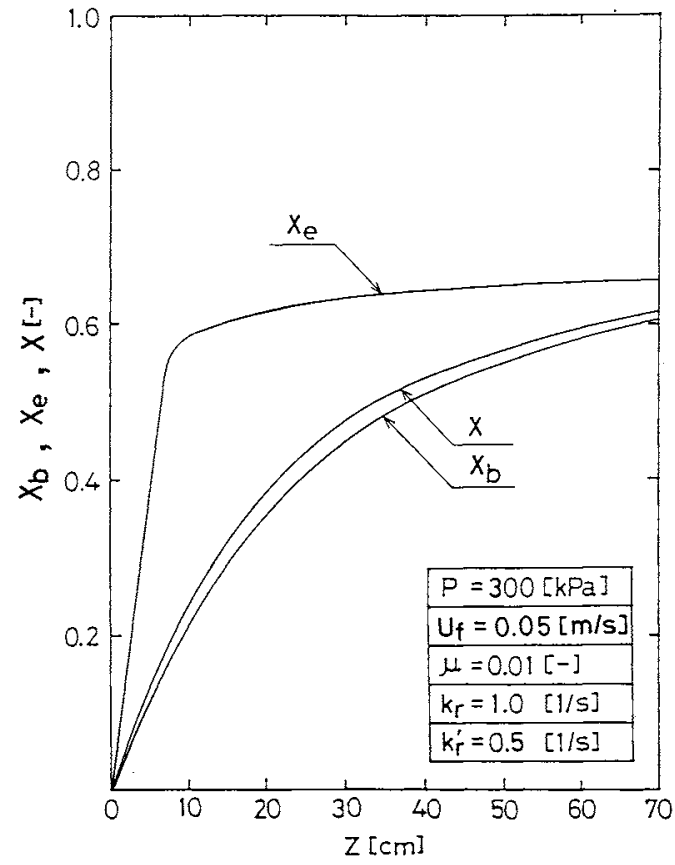

Fig. 10. Three kinds of conversion against $z$ by taking $u_{e}$, $\phi_{e}$ and $\varepsilon_{e m l}$ into account when an isothermal first-order reversible reaction is occurring (cracking catalyst, $k_{\mathrm{r}}=$ $1.0 \mathrm{~s}^{-1}, k_{r}^{\prime}=0.5 \mathrm{~s}^{-1}, \mu=0.01, P=300 \mathrm{kPa}, L_{\mathrm{s}}=0.62 \mathrm{~m}, u_{f}=$ $\left.0.05 \mathrm{~m} \cdot \mathrm{s}^{-1}\right)$

top of the bed as shown in Fig. 12. The chain line is shown as a reference when $u_{e} \phi_{e}$ is doubled.

The difference between the dotted and solid curves

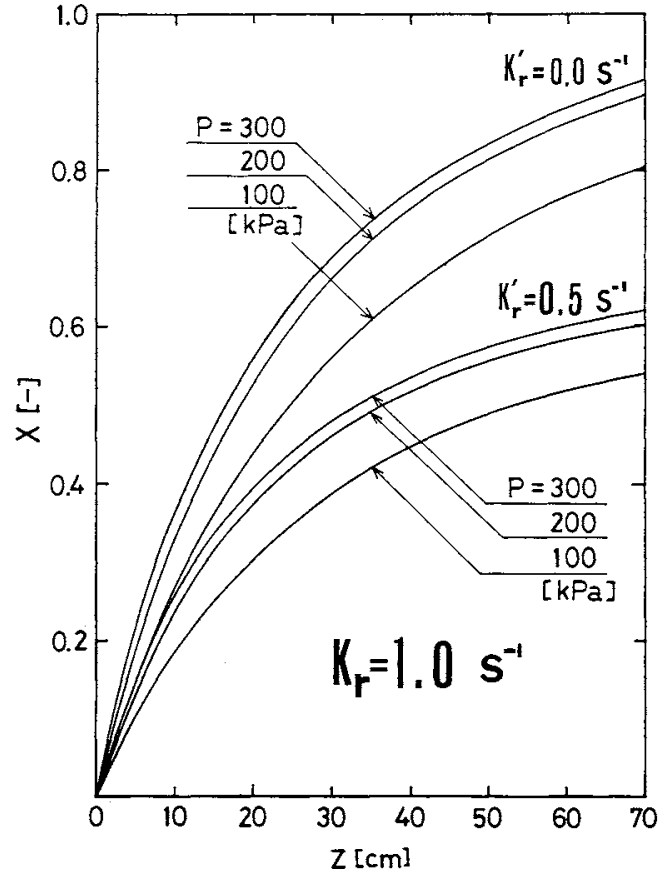

Fig. 11. Overall conversions when isothermal first-order irreversible or reversible reactions are occurring in a slightly pressurized fluidized bed (cracking catalyst, $\mu=0.01, P=100$, 200 and $300 \mathrm{kPa}, L_{\mathrm{s}}=0.62 \mathrm{~m}, u_{f}=0.05 \mathrm{~m} \cdot \mathrm{s}^{-1}$ )

in Fig. 12 might be small; however, the probable a little lower conversion would be substantial in a conversion estimation of industrial scale. 


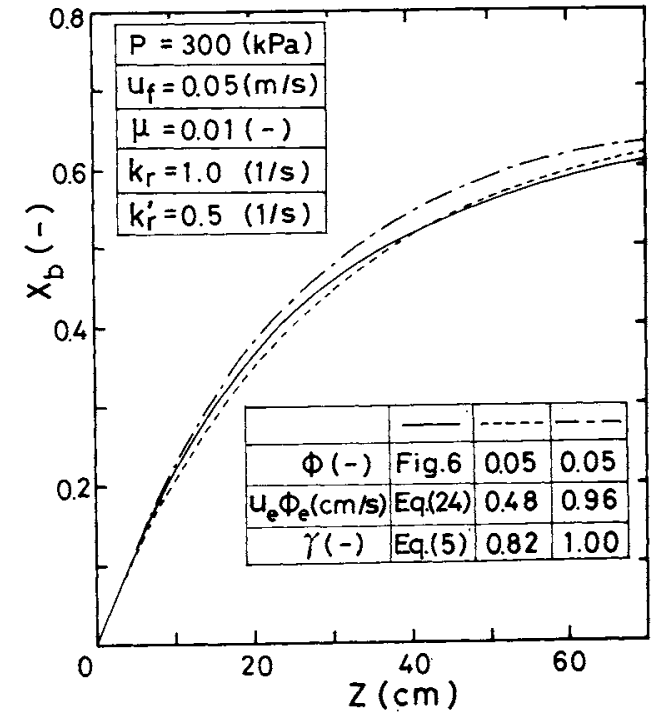

Fig. 12. Comparison of conversions of bubble phase by the conventional two-phase model and by the modified method proposed. The chain line is shown as a reference (cracking catalyst, $\mu=0.01, P=300 \mathrm{kPa}, L_{s}=0.62 \mathrm{~m}, u_{f}=0.05 \mathrm{~m} \cdot \mathrm{s}^{-1}$ )

\section{Conclusions}

A multi-channel light transmission method with a bubble elimination circuit has been developed for the analysis of emulsion phase in a fluidized bed of cracking catalyst. The spatial distributions of porosity of emulsion phase and bubble frequency showed a slight increase with pressure and these features of emulsion phase were found to be not so uniform throughout the bed, though it is axi-symmetrical as a whole.

Three kinds of conversion for isothermal first-order reaction were numerically examined by taking the porosity distribution and the fraction of emulsion phase into account and assuming that the reaction scheme and other characteristic values such as $k_{o b} a_{b} /$ $\phi_{b}, k_{r}, k_{r}^{\prime}$ and $\mu$ are independent of pressure.

As for the overall conversion at the top of the dense phase, the calculation showed a gain of approximately seven percent when the bed was pressurized from $100 \mathrm{kPa}$ to $300 \mathrm{kPa}$ under the assumptions stated above. A slight decrease in volume fraction of emulsion phase with pressure was found to be effective in increasing the overall conversion.

Conversion by the proposed method considering the physical properties of emulsion phase showed values a little lower than does the conventional twophase model at the upper part of the bed within the parameter values adopted.

\section{Acknowledgments}

The authors express their gratitude to the staff of the Workshop for Experimentation and Practice, School of Engineering, Nagoya University, for preparing pressure columns, dust removal and probe support systems. They also thank Ms. T. Itoh for typing this manuscript.

A part of the computation of this work was carried out at the Nagoya University Computation Center.

$$
\begin{aligned}
& \text { Nomenclature } \\
& A_{b}, A_{e} \quad=\text { reactant concentration in each phase } \quad[-] \\
& A_{b o}, A_{e o}=\text { inlet reactant concentration in each phase [-] } \\
& a_{b} \quad=\text { surface area of bubble phase } \\
& {\left[\mathrm{m}^{2} \text { (bubble) } \cdot \mathrm{m}^{-3} \text { (fluidized bed) }\right]} \\
& D_{T} \quad=\text { diameter of column }(=0.082) \quad[\mathrm{m}] \\
& \left.\begin{array}{lll}
\bar{d}_{p} \quad= & \text { fifty percent particle diameter in mass } \\
& \text { base distribution }
\end{array}\right] \\
& E_{a}, E_{i} \quad=\text { output of analog multiplexer [v] } \\
& E_{e}, E_{r} \quad=\text { output of analog switch [v] } \\
& \bar{E}_{e}, \bar{E}_{r} \quad=\text { output of integrator [v] } \\
& \begin{array}{lll}
f_{b} & =\text { frequency of bubbles } & {\left[\mathrm{s}^{-1}\right]} \\
\bar{f}_{b} & =\text { mean of } f_{b} & {\left[\mathrm{~s}^{-1}\right]}
\end{array} \\
& K \quad=\mathrm{k}_{r} / k_{r}^{\prime}
\end{aligned}
$$

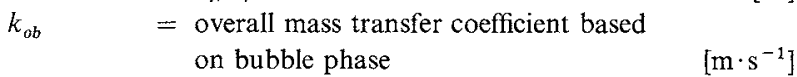

$$
\begin{aligned}
& \begin{aligned}
k_{r} \quad= & \text { rate constant of forward reaction based } \\
& \text { on fixed bed } \quad\left[\mathrm{s}^{-1}\right]
\end{aligned} \\
& \begin{aligned}
k_{r}^{\prime}= & \text { rate constant of backward reaction based } \\
& \text { on fixed bed }
\end{aligned} \\
& L_{f} \quad=\text { fluidized bed height of dense phase } \quad \text { [m] } \\
& L_{s} \quad=\text { static bed height [m] }
\end{aligned}
$$

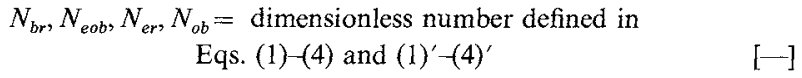

$$
\begin{aligned}
& P_{b}, P_{e} \quad=\text { product concentration in each phase } \quad[-] \\
& R C \quad=\text { integral time }(=33.3) \quad \text { [s] } \\
& r, r_{i} \quad=\text { radius } \quad[\mathrm{cm}] \text { or }[\mathrm{m}] \\
& S=\text { cross section of the bed }\left(=52.2 \times 10^{-4}\right) \quad\left[\mathrm{m}^{2}\right] \\
& s_{i} \quad=\text { element of } S(i=1-4) \quad\left[\mathrm{m}^{2}\right] \\
& T \quad=\text { total measuring term for a run }(=100) \text { [s] } \\
& T_{e} \quad=\text { total emulsion term } \quad[\mathrm{s}] \\
& u_{b}, u_{e} \quad=\text { local gas velocity through each phase } \quad\left[\mathrm{m} \cdot \mathrm{s}^{-1}\right] \\
& u_{f} \quad=\text { superficial gas velocity } \quad\left[\mathrm{m} \cdot \mathrm{s}^{-1}\right] \\
& u_{m b} \quad=\text { minimum bubbling velocity }\left(\doteqdot 3 u_{m f}\right) \quad\left[\mathrm{m} \cdot \mathrm{s}^{-1}\right] \\
& u_{m f} \quad=\text { minimum fluidization velocity } \\
& (=0.0016) \\
& X \quad=\text { weight of catalyst in the column }(=1.6) \quad[\mathrm{kg}] \\
& \begin{aligned}
X & \text { overall conversion defined by Eq. (18) } \\
& \text { or (21) } \\
X_{b}, X_{e}= & \text { conversion for each phase defined by }
\end{aligned} \\
& \text { Eqs. (16), (17) or (19), (20) } \\
& Z \quad=z / L_{f} \quad[-] \\
& z \quad=\text { height above the distributor } \quad[\mathrm{cm}] \text { or }[\mathrm{m}] \\
& \alpha, \beta, \delta \quad=\text { parameters defined in Table } 1 \quad[-] \\
& \gamma \quad=\text { correction factor that reduces emulsion phase } \\
& \text { to its equivalent fixed bed, defined by } \\
& \text { Eq. (5) } \\
& =\text { porosity of emulsion phase } \\
& =\text { mean of } \varepsilon_{e m l} \text { at } r=r_{i} \\
& =\text { ensemble average defined by Eq. (32) or } \\
& \text { area weighted mean of } \bar{\varepsilon}_{e m l} \\
& =\text { porosity of emulsion phase at minimum } \\
& \text { bubbling fluidization }(=0.48) \\
& =\text { porosity of emulsion phase at minimum } \\
& \text { fluidization }(=0.43) \\
& =\text { porosity of cracking catalyst in fixed } \\
& \text { bed }(=0.365) \\
& =\text { angle of probe rotation } \\
& \begin{aligned}
\kappa \quad= & \text { constant defined by Eq. (27); ranges from } \\
& 4.0 \text { to } 6.0 \text { for seven sensors }\left[\mathrm{m}^{3} \cdot \mathrm{kg}^{-1}\right]
\end{aligned} \\
& \begin{aligned}
\kappa \quad= & \text { constant defined by Eq. (27); ranges from } \\
& 4.0 \text { to } 6.0 \text { for seven sensors }\left[\mathrm{m}^{3} \cdot \mathrm{kg}^{-1}\right]
\end{aligned} \\
& {[-]} \\
& {[-]} \\
& \text { [-] } \\
& \text { [-] } \\
& \text { [-] }
\end{aligned}
$$




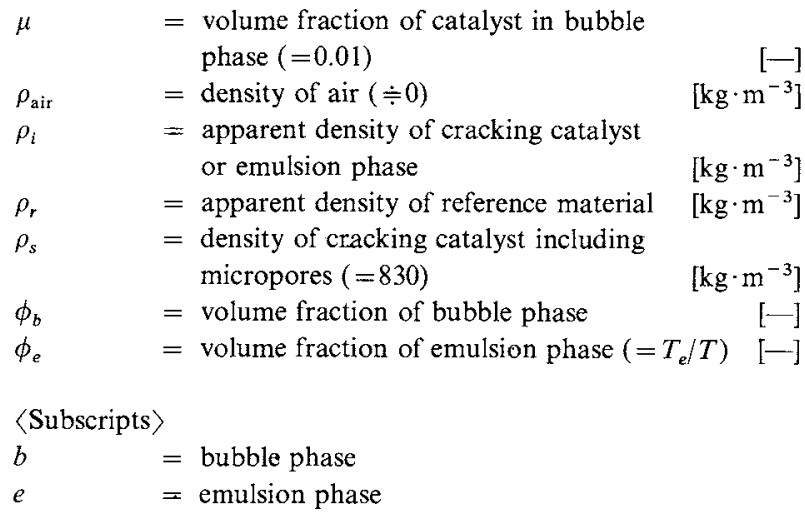

\section{Literature Cited}

1) Barreto, G. F., J. G. Yates and P. N. Rowe: Chem. Eng. Sci., 38, 1935 (1983).

2) Chitester, D. C., R. M. Kornosky, L. S. Fan and J. P. Danko: Chem. Eng. Sci., 39, 253 (1984).

3) Fukuta, K., M. Yamazaki and Y. H. Li: Preprints of the 19th Autumn Meeting, Nagoya, Soc. Chem. Engrs., Japan, p. 522 (1985).

4) Funabashi, Y., M. Yamazaki and G. Jimbo: Preprints of the 16 th Autumn meeting, Toyota, Soc. Chem. Engrs., Japan, p. 174 (1982).

5) Kawabata, J., M. Yumiya, Y. Tazaki, S. Honma, T. Chiba, T. Sumiya and K. Endo: J. Chem. Eng. Japan, 14, 85 (1981).

6) Kawanishi, K. and M. Yamazaki: Proc. 4th Int. Conf. on
Fluidization, Kashikojima, Japan, p. 37, Engineering Foundation (1983).

7) Weimer, A. W. and G. J. Zuarderer: $A I C h E J ., 31,1019$ (1985).

8) Yamazaki, M. and K. Fukuta: to be submitted.

9) Yamazaki, M., K. Fukuta and Y. H. Li: Preprints of the 18th Autumn Meeting, Fukuoka, Soc. Chem. Engrs., Japan, p. 49 (1984).

10) Yamazaki, M., K. Fukuta and J. Tokumoto: Proc. of the World Congress III of Chemical Engineering, Tokyo, Vol. 3; p. 433 (1986).

11) Yamazaki, M. and T. Miyauchi: J. Chem. Eng. Japan, 4, 324 (1971).

12) Yamazaki, M. and T. Miyauchi: J. Chem. Eng. Japan, 6, 285 (1973).

13) Yamazaki, M., T. Sugino and G. Jimbo: Preprints of the 43rd Annual Meeting, Nagoya, Soc. Chem. Engrs., Japan, p. 54 (1978).

14) Yang, W. C., D. C. Chitester, R. M. Kornosky and D. L. Keairns: AIChE J., 31, 1086 (1985).

15) Yue, P. L., L. Rizzuti and V. Augugliaro: Chem. Eng. Sci., 41, $171(1986)$

(Presented in part at the 51st Annual Meeting of the Society of Chemical Engineers, at Osaka, March, 1986; at the World Congress III of Chemical Engineering, at Tokyo, September, 1986; and at the 52nd Annual Meeting of the Society of Chemical Engineers, at Nagoya, April, 1987.) 